Hydrology and Earth System Sciences, 9, 127-137, 2005

www.copernicus.org/EGU/hess/hess/9/127/

SRef-ID: $1607-7938 /$ hess/2005-9-127

European Geosciences Union

\title{
Empirical Mode Decomposition in 2-D space and time: a tool for space-time rainfall analysis and nowcasting
}

\author{
S. Sinclair and G. G. S. Pegram \\ Civil Engineering, University of KwaZulu-Natal, Durban, South Africa
}

Received: 23 December 2004 - Published in Hydrology and Earth System Sciences Discussions: 10 February 2005

Revised: 20 May 2005 - Accepted: 12 July 2005 - Published: 22 July 2005

\begin{abstract}
A data-driven method for extracting temporally persistent information, at different spatial scales, from rainfall data (as measured by radar/satellite) is described, which extends the Empirical Mode Decomposition (EMD) algorithm into two dimensions. The EMD technique is used here to decompose spatial rainfall data into a sequence of high through to low frequency components. This process is equivalent to the application of successive low-pass spatial filters, but based on the observed properties of the data rather than the predetermined basis functions used in traditional Fourier or Wavelet decompositions. It has been suggested in the literature that the lower frequency components (those with large spatial extent) of spatial rainfall data exhibit greater temporal persistence than the higher frequency ones. This idea is explored here in the context of Empirical Mode Decomposition. The paper focuses on the implementation and development of the two-dimensional extension to the EMD algorithm and it's application to radar rainfall data, as well as examining temporal persistence in the data at different spatial scales.
\end{abstract}

\section{Introduction}

Spatial rainfall data contain information at a broad range of spatial scales (Schertzer and Lovejoy, 1987; Harris et al., 2001; Pegram and Clothier, 2001). It has been suggested in the literature (Seed, 2003; Turner et al., 2004) that the lower frequency components exhibit more temporal persistence than the higher ones; this premise is used here to prepare the data for nowcasts based on the evolution of the lower frequency components of space-time rainfall sequences. Examination of the (radially averaged) power spectrum (Fig. 1b) derived from a typical instantaneous estimate

Correspondence to: S. Sinclair

(sinclaird@ukzn.ac.za) of rainfall rate obtained by weather radar (Fig. 1a) indicates that most of the power, hence potential for deterministic prediction in the context of nowcasting, is contained in the low frequency components.

In this paper we focus on a data-driven technique to extract the high frequency (less persistent in time) modes as the first step in a rainfall nowcasting scheme. The technique employed is a two-dimensional (2-D space) generalization of the one-dimensional Empirical Mode Decomposition (EMD) technique introduced by Huang et al. (1998). In a single dimension, EMD analysis produces a set of Intrinsic Mode Functions (IMFs) that are very nearly orthogonal; in two dimensions a set of Intrinsic Mode Surfaces (IMSs) is produced with similar quasi-orthogonal properties. Twodimensional EMD appears to have been first introduced by Linderhed (2002) in the context of image compression; the key contribution in this paper is to introduce the concept of 2-D EMD to the Hydrometeorological literature as a tool for the analysis of space-time rainfall data. This paper focuses on the implementation and development of the twodimensional extension of the EMD algorithm in this context, decomposing spatial rainfall data into its intrinsic spatial scale components.

In the application presented here, the least persistent IMS (exhibiting the highest local spatial frequency and least amount of spatial correlation - hence nearly white noise) is computed and removed from the raw rainfall data leaving a residual composed of the more persistent low frequency structural components in the data. This process is equivalent to using a low-pass spatial filter, based on the observed properties of the data rather than the predefined basis functions used in traditional Fourier or Wavelet scale decompositions. In Sects. 2 and 3, simple theoretical examples, showing the power of EMD in one and two dimensions, are presented as a "proof of concept" before applying the procedure to observed radar rainfall data from Bethlehem, South Africa (Sect. 4). These complement and extend the original presentation by

(C) 2005 Author(s). This work is licensed under a Creative Commons License. 


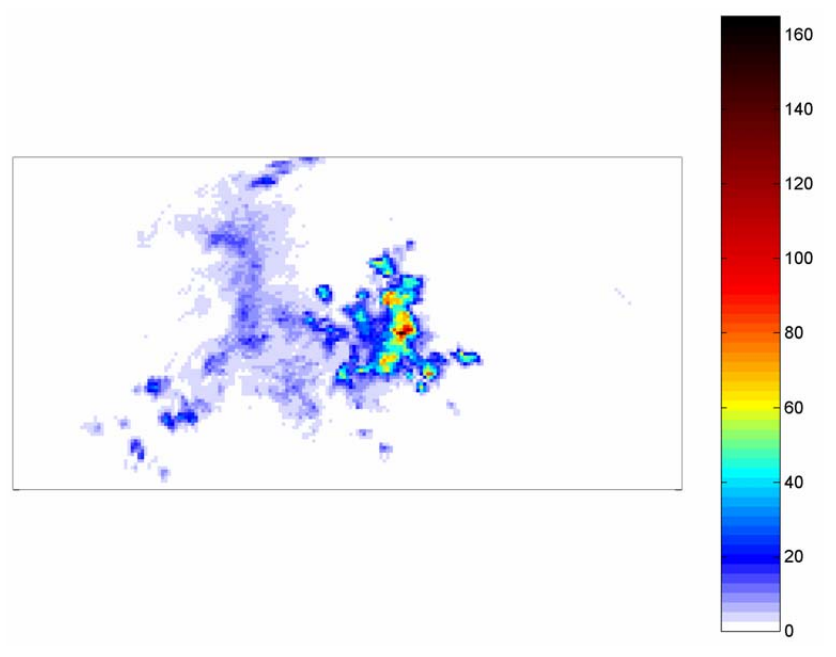

Fig. 1. (a) An observed convective rainfall field measured by SBand weather radar at Bethlehem, South Africa (colour scale indicates instantaneous rain rate in $\mathrm{mm} / \mathrm{h}$ ). The image is $100 \times 200$ with $1 \mathrm{~km}^{2}$ pixels.

Huang et al. (1998) and Flandrin et al. (2004). Computational aspects relating to image processing and surface fitting are covered in detail (Sect. 4) and conclusions are drawn in Sect. 5.

\section{Empirical Mode Decomposition in a single dimension}

The basic idea embodied in the EMD analysis, as introduced by Huang et al. (1998), is to allow for an adaptive and unsupervised representation of the intrinsic components of linear and non-linear signals based purely on the properties observed in the data without appealing to the concept of stationarity. As Huang et al. (1998) point out in their abstract: "This decomposition method is adaptive and therefore highly efficient. Since the decomposition is based on the local characteristic time scale of the data, it is applicable to nonlinear and non-stationary processes."

Few sequences of observations of natural phenomena are long enough to test the hypothesis of stationarity and frequently, the phenomena are patently non-stationary. This tacitly applies in the measurement of rainfall at a point or in space-time because sequences of rain are interspersed with dry periods and during the raining periods, the variability of the intensity due to mixtures of rainfall type (stratiform, convective, frontal) confound the stationarity definition. The EMD algorithm copes with stationarity (or the lack of it) by ignoring the concept, embracing non-stationarity as a practical reality. For a fuller discussion of the genesis of these ideas, see the Introduction of Huang et al. (1998), who also heuristically demonstrate the implicit orthogonality of the sequences of Intrinsic Mode Functions (IMFs) defined by the EMD algorithm.

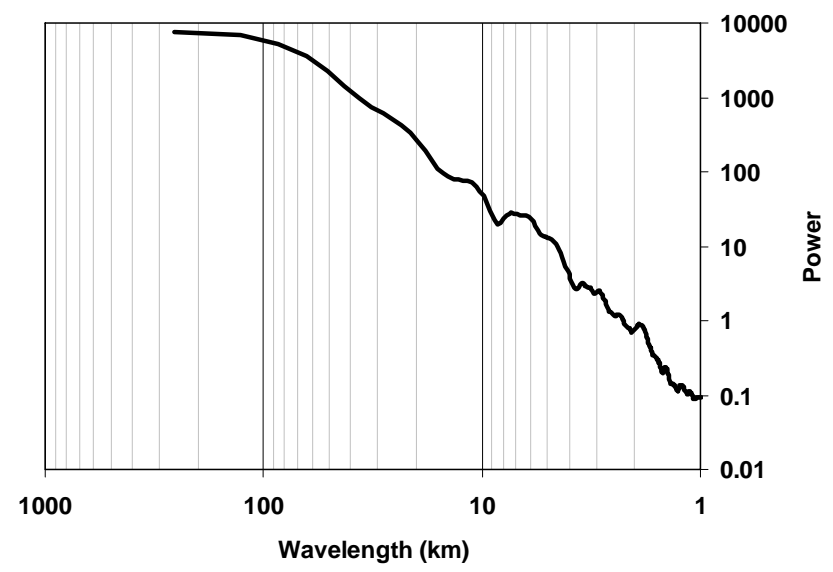

Fig. 1. (b) Radially averaged power spectrum of instantaneous rainfall rate from typical radar rainfall data shown in (a).

In the application of the EMD algorithm, the possibly nonlinear signal, which may exhibit varying amplitude and local frequency modulation, is linearly decomposed into a finite number of (zero mean) frequency and amplitude modulated signals, as well as a residual function which exhibits a single extremum, is a monotonic trend or is simply a constant. Although EMD is a relatively new data analysis technique, its power and simplicity have encouraged its application in a myriad of fields. It is beyond the scope of this paper to give a complete review of the applications, however a few interesting examples are cited here to give the reader a feeling for the broad scope of applications. Chiew et al. (2005) examine the one-dimensional EMD of several annual streamflow time series to search for significant trends in the data, using bootstrapping to test the statistical significance of identified trends. The technique has been used extensively in the analysis of ocean wave data (Huang et al., 1999; Hwang et al., 2003) as well as in the analysis of polar ice cover (Gloersen and Huang, 2003). EMD has also been applied in the analysis of seismological data by Zhang et al. (2003) and has even been used to diagnose heart rate fluctuations (Balocchi et al., 2004).

\subsection{Computing the one-dimensional EMD}

The EMD algorithm extracts the oscillatory mode that exhibits the highest local frequency from the data ("detail" in the Wavelet context or the result of a high-pass filter in Fourier analysis), leaving the remainder as a "residual" ("approximation" in Wavelet analysis). Successive applications of the algorithm on the sequence of residuals produce a complete decomposition of the data. The final residual is a constant, a monotone trend or a curve with a single extremum.

The EMD of a one-dimensional data set $z(k)$ is obtained using the following procedure:

1. Set $r_{0}(k)=z(k)$ and set $i=1$. 


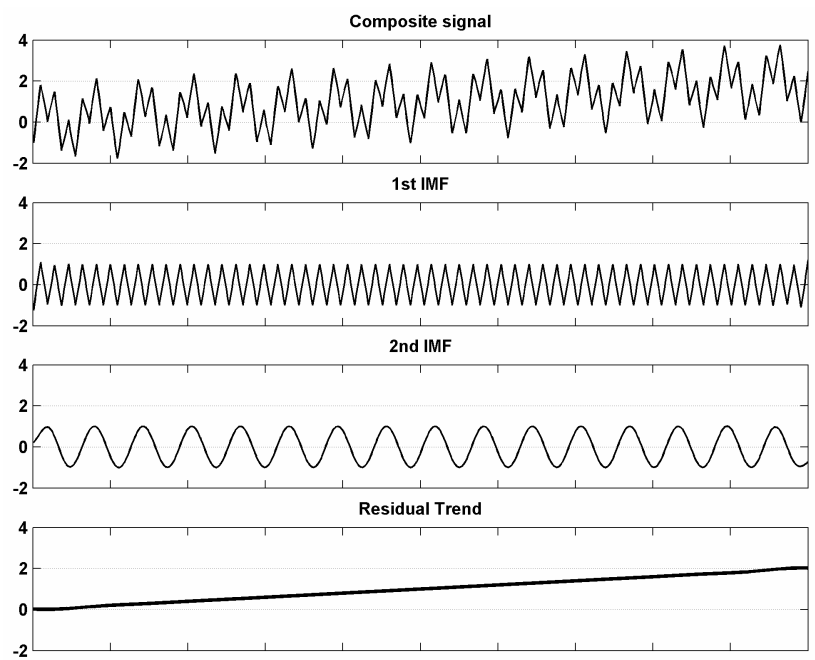

Fig. 2. EMD based signal separation; all IMFs are plotted to the same vertical scale. Top panel is the combined signal; lower 3 panels are the decomposition which recaptures, almost exactly, the original components.

2. Identify all of the extrema (maxima and minima) in $r_{i-1}(k)$.

3. Compute a maximal envelope, $\max _{i-1}(k)$, by interpolating between the maxima found in step 2. Similarly compute the minimal envelope, $\min _{i-1}(k)$. Cubic splines (as suggested by Huang et al., 1998) appear to be the most appropriate interpolation method for deriving these envelopes in one dimension (Flandrin et al., 2004).

4. Compute the mean value function of the maximal and minimal envelopes $m_{i-1}(k)=\frac{\left[\max _{i-1}(k)+\min _{i-1}(k)\right]}{2}$.

5. The estimate of the IMF is computed from $\operatorname{IMF}_{i}(k)=r_{i-1}(k)-m_{i-1}(k)$.

Each IMF is supposed to oscillate about a zero mean and in practice it is necessary to perform a "sifting" process by iterating steps $2-5$ (setting $r_{i-1}=\mathrm{IMF}_{i}$ before each iteration) until this is achieved.

6. Once the $\mathrm{IMF}_{i}$ has a mean value that is sufficiently close to zero over the length of the data (defined by a stopping criterion within some predefined tolerance $\varepsilon$ ) the residual $r_{i}(k)=r_{i-1}(k)-\mathrm{IMF}_{i}(k)$ is computed. Alternatively the sifting procedure can be stopped when the difference in the standard deviation of successive estimates of $\mathrm{IMF}_{i}$ falls below a critical threshold (Huang et al., 1998).

7. If the residual $r_{i}(k)$ is a constant or trend then stop; else increment $i$ and return to step 2 .

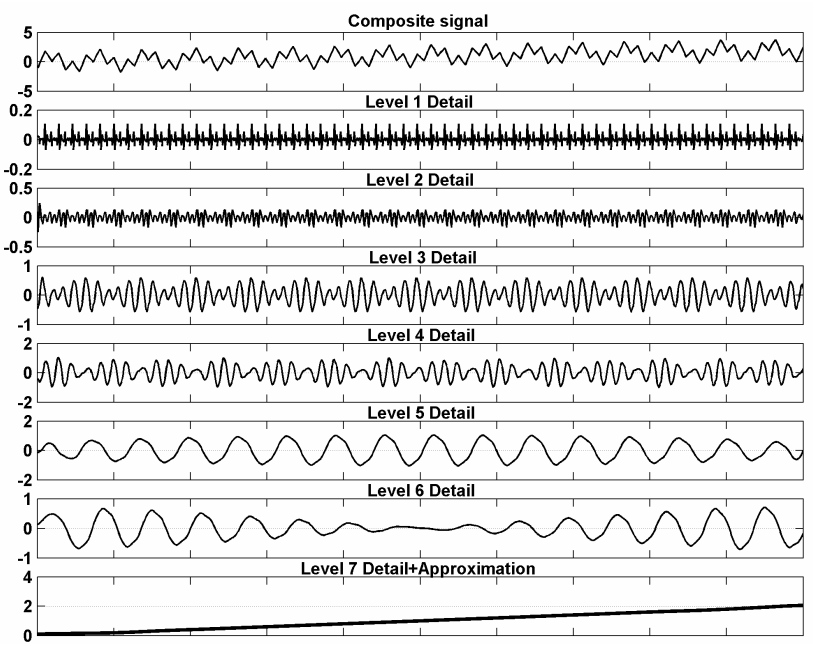

Fig. 3. Wavelet based signal separation - The "data" are the same as in Fig. 2, the vertical scale has been compressed for a compact presentation. An arbitrarily chosen db5 wavelet basis has been used.

Figure 2 shows the EMD of a composite data series (shown in the first panel) that is the summation of a sine wave, a triangular waveform and a slowly varying trend. The compact representation obtained by EMD extracts (almost perfectly except near the ends) the three separate time series (shown in panels 2 to 4 ) that make up the composite signal, without resorting to Fourier or Wavelet techniques with restrictive assumptions about the form of the underlying oscillatory modes or basis functions. Figure 3 shows the analysis of the same data, using Wavelet decomposition. Here a fifth order Daubechies wavelet basis was (arbitrarily) chosen for illustration purposes; this choice of basis function may not be optimal for detrending but serves to demonstrate a typical decomposition. Seven levels of decomposition were required before the trend became apparent; this decomposition is clearly far less compact and physically meaningful than the EMD results in this case.

A similar decomposition analysis can be carried out using Fourier techniques. The Discrete Fourier approximation of a signal can be defined in terms of the Euler-Fourier coefficients $\left(a_{0}, a_{k}, b_{k}\right)$ with $k=1,2, \ldots, m$ (Eq. 1). The coefficients are all that are required to reconstruct the series and any signal can be well approximated (as long as it satisfies the Dirichlet conditions), provided $m$ is sufficiently large. In Eq. (1), $F\left(x_{j}\right)$ is the Fourier approximation of the signal $y_{j}$ at each of the $n$ discrete (evenly spaced) data points $x_{j}$. L is the range of values $x_{j}$ over which the data set is assumed periodic.

$$
\begin{aligned}
& F\left(x_{j}\right)=\frac{a_{0}}{2}+\sum_{k=1}^{m}\left\{a_{k} \cos \left(2 \pi k x_{j} / L\right)+b_{k} \sin \left(2 \pi k x_{j} / L\right)\right\}(1) \\
& a_{k}=2 \sum_{j=1}^{n} \cos \left(2 \pi k x_{j} / L\right) y_{j} / n
\end{aligned}
$$




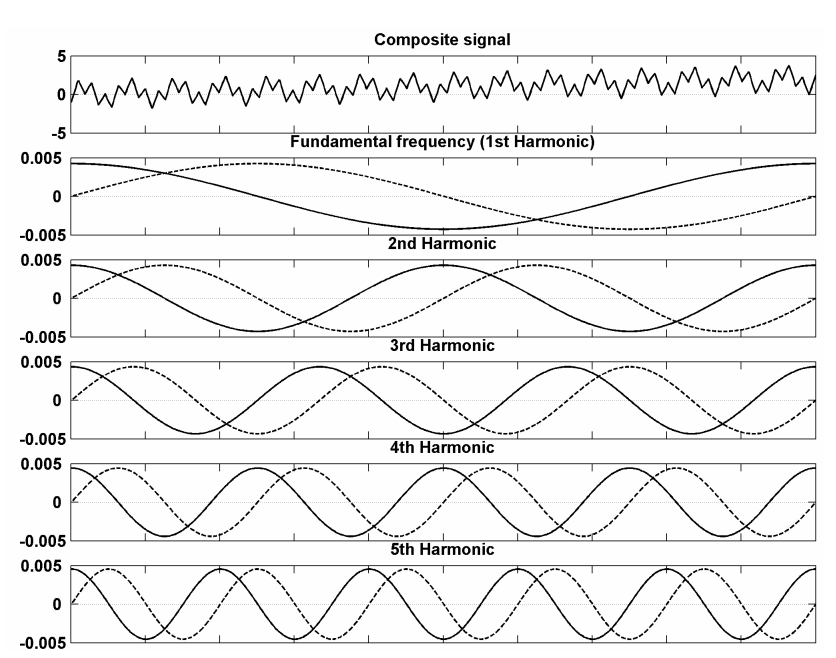

Fig. 4. Fourier based signal separation, the first 5 of 75 components - The dashed lines show the sine component and the solid lines the cosine component.

$b_{k}=2 \sum_{j=1}^{n} \sin \left(2 \pi k x_{j} / L\right) y_{j} / n$.

Figures 4 and 5 show the result of decomposing the data using a finite Fourier series. Figure 4 shows the first 5 harmonics; while Fig. 5 shows the series reconstruction by accumulating the lower harmonics up to $m$. Computing the Euler-Fourier coefficients provides a compact approximation of the original signal but fails to extract physically meaningful information. The ability to determine meaningful structural information is clearly important in a nowcasting context, which cannot be bound by the periodicity assumption implicit in Fourier methods.

\section{Empirical Mode Decomposition in two dimensions}

In two dimensions the EMD process is conceptually the same as for a single dimension, except that the curve fitting exercise becomes one of surface fitting and the identification of extrema becomes (a little) more complicated. Very little work appears to have been done which applies the EMD technique to two-dimensional data. Han et al. (2002) use EMDs in one dimension along four different directions to smooth Synthetic Aperture Radar (SAR) images and remove speckle. Nunes et al. (2003) develop a technique, which they term "Bidimensional Empirical Mode Decomposition" (BEMD) in the context of texture analysis in image data where they demonstrate several examples of intrinsic mode extraction from image data. Linderhed $\left(2002,2004^{1}\right)$ examined the use of EMD in two dimensions for image compression. Both of these implementations are very similar to what we propose in this paper. The 2-D EMD provides a truly two-

\footnotetext{
${ }^{1}$ http://www.icg.isy.liu.se/ anna/emd-samp.pdf
}

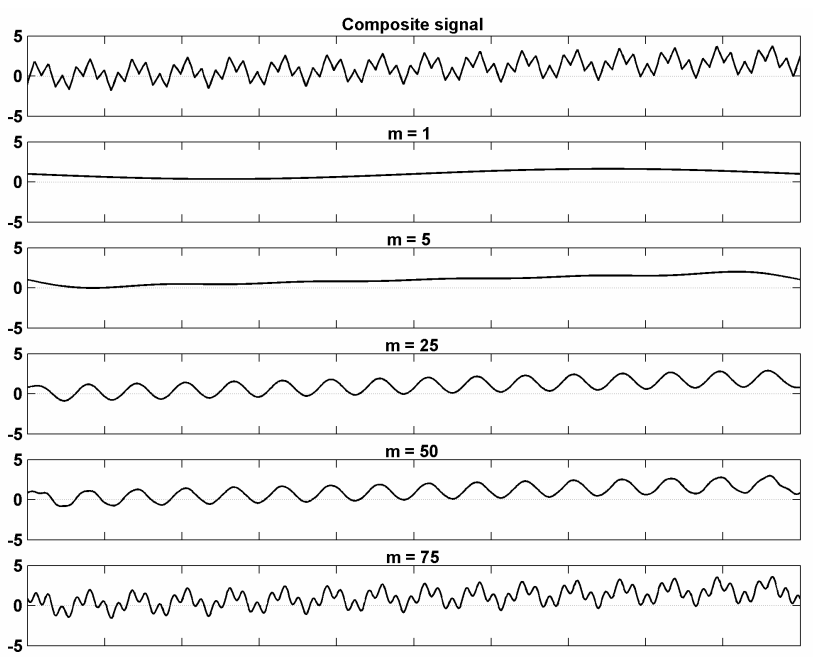

Fig. 5. Reconstruction of the signal from the sine and cosine components, $m$ represents the number of Euler-Fourier coefficients used in each reconstruction.

dimensional analysis of the intrinsic oscillatory modes inherent in the data. Two-dimensional Fourier and Wavelet analyses are really applications of their one-dimensional counterparts in a number of principal directions. Fourier analysis concentrates on orthogonal "East-West" and "North-South" directions (e.g. Press et al., 1992). Wavelet analysis can, in general, consider any direction of the wavelet relative to the data, however a typical 2-D Wavelet analysis examines only horizontal, vertical and diagonal orthonormal wavelet basis functions (Daubechies, 1992, pp. 313; Kumar and FoufoulaGeorgiou, 1993). In contrast, EMD produces a fully twodimensional decomposition of the data, based purely on spatial relationships between the extrema, independent of the orientation of the coordinate system in which the data are viewed.

\subsection{Description of the algorithm}

The algorithm follows intuitively from the one-dimensional case and may be briefly summarised as follows:

1. Locate the extrema in the 2D space including maximal and minimal plateaus.

2. Generate the bounding envelopes using appropriate surface fitting techniques. We suggest conical Multiquadrics (for reasons explained in Sect. 3.2).

3. Compute the mean surface function as the average value of the upper and lower envelopes.

4. Determine the first estimate of an IMS by subtracting the mean surface from the data.

5. Iterate until the IMS mean surface function is close to zero everywhere. 

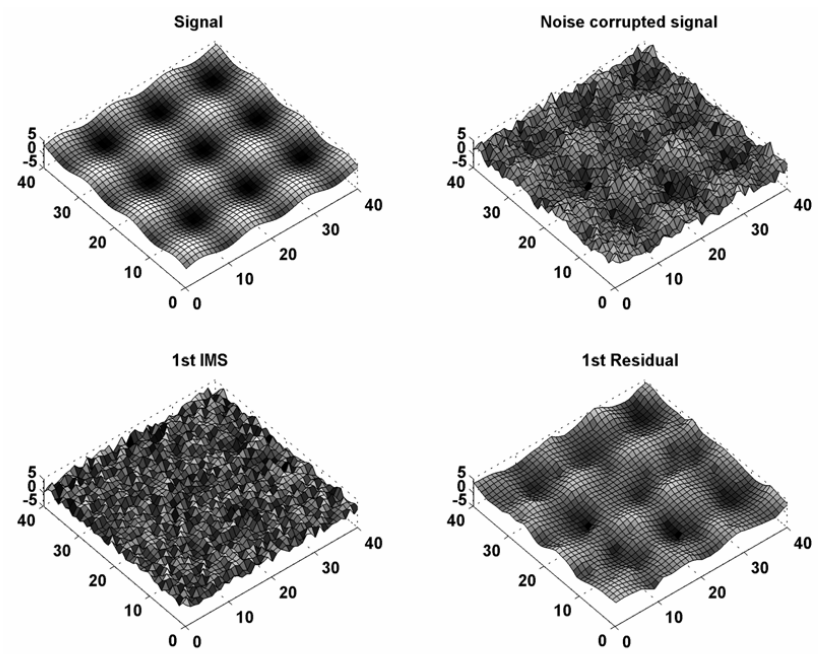

Fig. 6. Example of EMD used for noise removal on a 2-D sine wave. The bulk of the additive white noise in the corrupted signal is well captured by the first IMS.

\section{Estimate the IMS and Residual.}

7. If the Residual is a constant or a monotone trend, then stop; else return to step 2.

\subsection{Surface fitting for extremal envelope generation}

The generation of maximal and minimal envelopes is of key importance to a successful 2-D EMD implementation and is the most computationally intensive task. The problem is a familiar one of collocating a smooth surface to randomly scattered data points in two-dimensions. There are several options available to achieve this. Ultimately the fitting procedure reduces to computing the unknown value of the surface at a point $s_{i}=\left(x_{i}, y_{i}\right)$, by some linear (or nonlinear) weighting of the known data. In general, a basis function determines the influence of each known data point based on its spatial position relative to the unknown point $s_{i}$. Nunes et al. (2003) use radial basis functions while Linderhed uses bi-cubic splines (Linderhed, 2002) and later chooses the more suitable option of Thin Plate Splines (Linderhed, 2004). We use radial basis functions (technically, conical Multiquadrics), which are identical to Kriging (Cressie, 1991) with a purely linear semi-variogram model. It could perhaps be argued that it would be more appropriate to fit a semi-variogram model to the maxima and minima, but we feel this would be overelaborate and presumptuous, as the extrema are only related by distance and cannot be considered drawn from a stationary correlated random field. Invoking Occam's razor in the spirit of Huang's original derivation of EMD, we wish to let the data do the talking and conical Multiquadrics assume the least structure of any linear surface fitting algorithm.
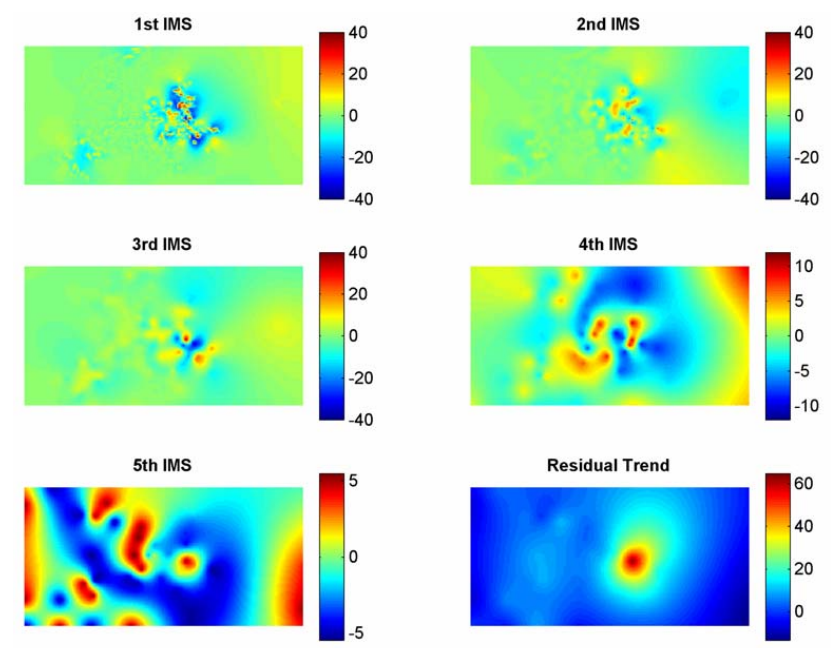

Fig. 7. Naïve EMD of the observed rainfall field shown in Fig. 1a note the change in scale of the rain rates in the IMSs.

The Ordinary Kriging estimate $\hat{z}_{i}$ at any point $i$ based on $n$ observed data points is

$\hat{z}_{i}=\sum_{k=1}^{n} \lambda_{k} z_{k}$

where $z_{k}$ are the observations and $\lambda_{k}$ are weights associated with each observation and the target point. The mean is assumed unknown and the weights $\lambda_{k}$ are constrained to sum to unity. The vector of weights $\lambda$ is obtained by solving the linear system in Eq. (5)

$\left[\begin{array}{cc}\Gamma & u \\ u^{T} & 0\end{array}\right]\left[\begin{array}{l}\lambda \\ \mu\end{array}\right]=\left[\begin{array}{l}\gamma \\ 1\end{array}\right]$

where $\gamma$ is a vector of semivariogram values, in this application simply defined by the linear distance basis function $\gamma\left(s_{i j}\right)=\left|s_{i j}\right|$ with $s_{i j}$ the distance between point $i$ and the $j=1,2, \ldots, n$ observation locations. $\Gamma$ is the matrix of distances between the observations, $\boldsymbol{u}$ is a vector of $n$ ones and $\mu$ is a Lagrange multiplier ensuring that the Kriging weights $\lambda_{k}$ sum to unity, as required. The solution of Eq. (5) is obtained using Singular Value Decomposition (SVD) in this application to ensure that a stable solution is assured (when the matrix is ill conditioned). This is achieved by truncating singular and near-singular components. Although SVD is computationally less efficient than (for example) LU decomposition as a means of solving a dense linear system, it's use is preferred here because of it's robustness in the face of the near-singular Kriging systems which are frequently encountered in gridded data applications (Wesson and Pegram, 2004).

A more efficient choice of interpolation technique would be useful and more work could be done in this regard, however care is required. Moving-neighbourhood Kriging (a possible alternative to reduce the number of control points) 


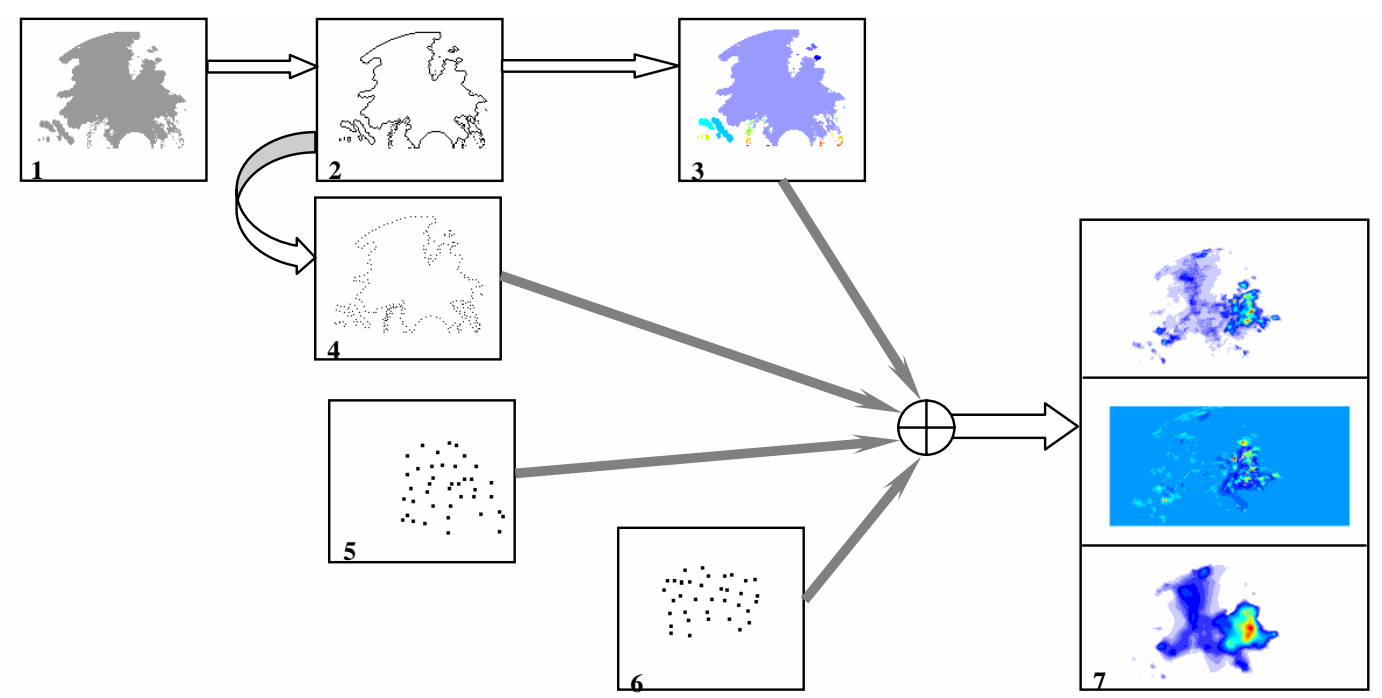

Fig. 8. Summary of data processing 1 . Mask the wet and dry areas; 2 . Trace the boundary of each wet region; 3 . Separately label each wet region; 4. Decimate the fence by a factor of 5, isolating the "fence posts"; 5. Isolate the maxima in each sub-region; 6 . Isolate the minima in each sub-region; 7. EMD analysis decomposes the data into the first IMS and the first residual using the maximal and minimal envelopes defined using the points in 4,5 and 6 .

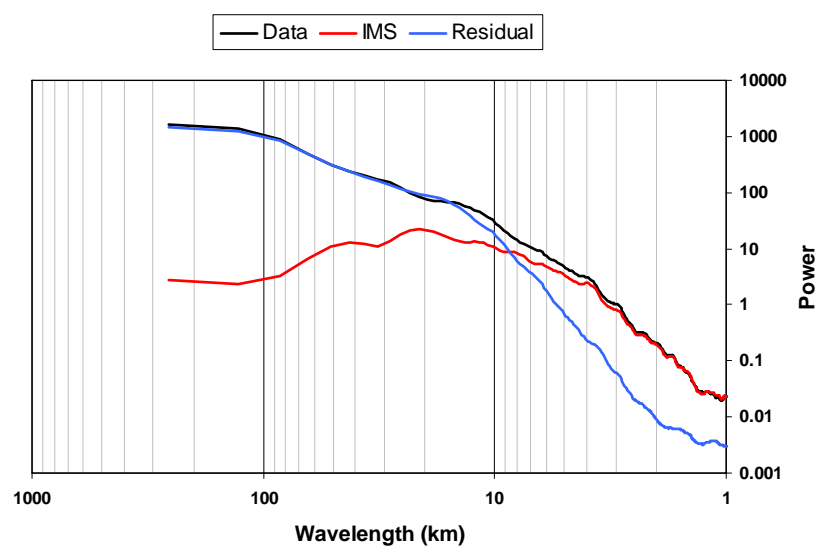

Fig. 9. Comparison of individual radially-averaged power spectra of the radar rainfall data (of Fig. 1a) with its EMD components: the first IMS and the first residual.

can produce unwanted discontinuities in regions that are data sparse (Chiles and Delfiner, 1999, pp. 201), such discontinuities would be amplified through the EMD sifting process. In addition, the particular choice of Ordinary Kriging as a method of generating the bounding envelopes was (partially) directed by the property that the estimates decay asymptotically to the mean of the observed extrema.

\subsection{Simple two-dimensional EMDs}

In this section, applications of the 2-D EMD technique are presented. As an artificially constructed example Fig. 6 shows the successful removal of noise added to a syntheti-

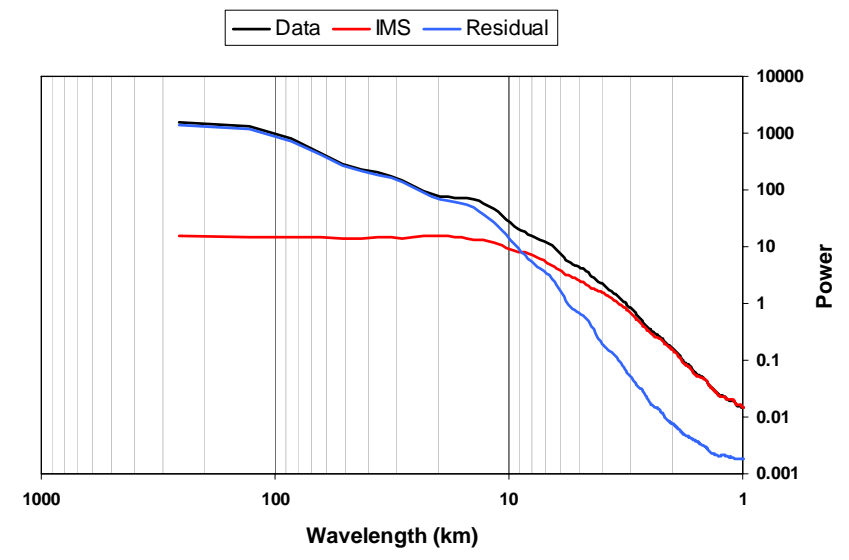

Fig. 10. The same as Fig. 9 but for the mean of individual power spectra for five consecutive, radar scans - Beginning with the spectra shown in Fig. 9.

cally generated two-dimensional sine signal. The noise (with it's high local spatial frequency) is almost completely described by the first IMS leaving a residual, which is closely representative of the underlying signal.

Turning to a realistic example of the type we have been aiming for, Fig. 1a showed an instantaneous radar rainfall field with an area of $100 \times 200 \mathrm{~km}$. A complete EMD of this field is shown in Fig. 7 using a direct application of the 2-D EMD process described in Sect. 3.1; note the change in scale of the individual IMSs. The final residual (with a single extremum) gives a clear indication of the position of the largest convective raincell evident in the field. 

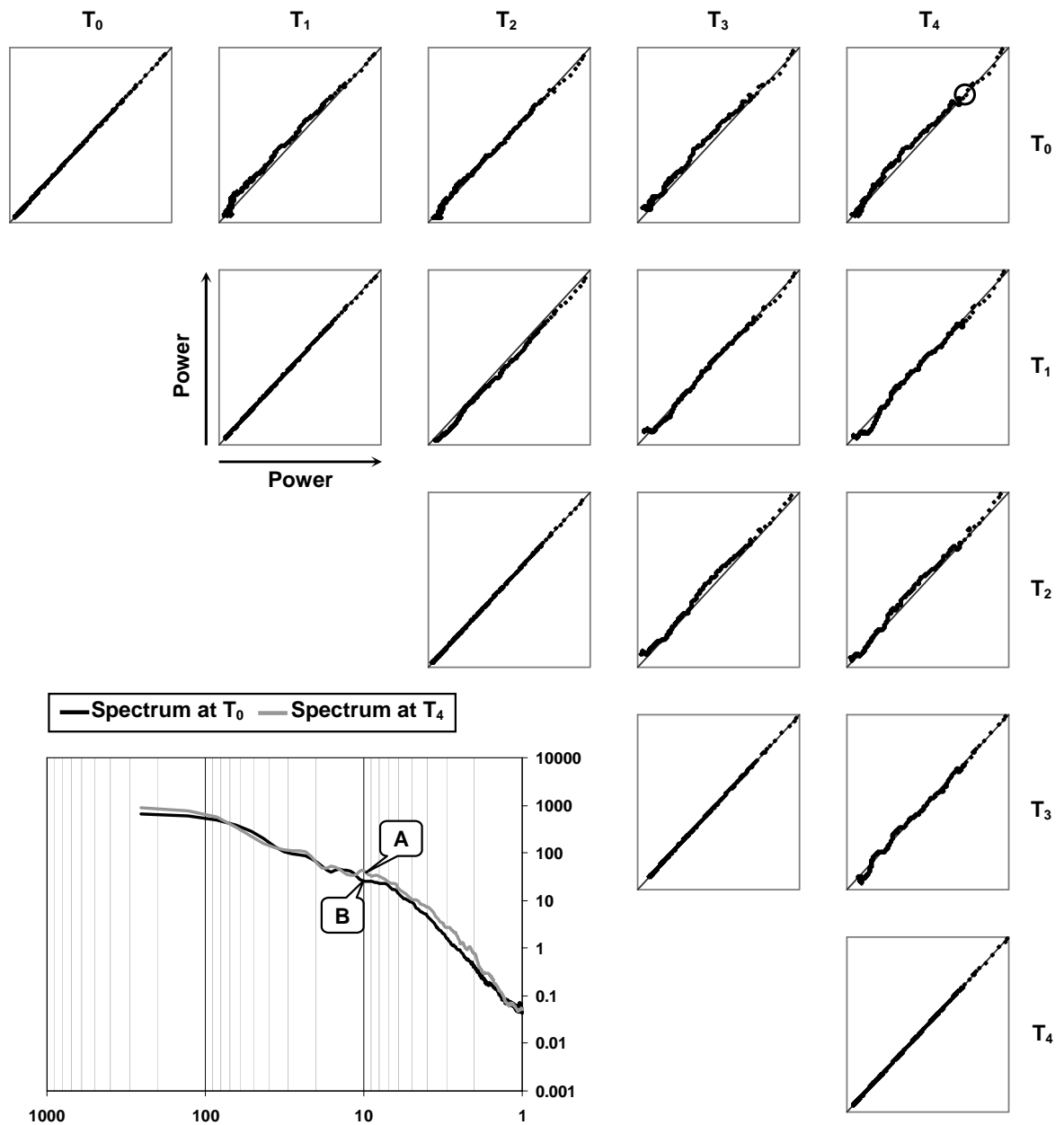

Fig. 11. Spectral persistence scatter plots of the original data for a sequence of rainfall fields and those at successive intervals. This is constructed by plotting the values of power for each field at corresponding wavelengths coaxially. For example points $A$ and $B$ at the $10 \mathrm{~km}$ wavelength are plotted against each other and appear ringed in the upper right diagram.

\section{Application of 2-D Empirical Mode Decomposition to rainfall data}

The simple 2-D EMD application presented in the previous section is computationally burdensome when applied to rainfall data. In this section, to overcome this drawback, a number of specific refinements are presented which combine to make EMD tractable in practical real-time situations.

\subsection{Image processing techniques and optimisations}

Since an application of 2-D EMD requires the use of surface fitting techniques, large linear systems must be solved. The size of a system is determined by the number of known data points which are to be used in combination to find the unknown values of the surface at each remaining position in the field. The highly variable nature of rainfall data means that the field contains a large number of extrema from which the bounding envelopes must be constructed. Additionally there are a large number of zero (no rain) data, which constitute minima. By only considering raining areas, the size of the linear systems requiring a solution are greatly reduced since each raining area (if more than one exists) will contain a considerably smaller number of extrema than the entire data region and each can be treated separately. Furthermore, it makes no sense to consider an EMD in areas where the variable of interest does not exist, in this case the areas that are not raining.

A number of well-known image processing techniques are implemented to isolate and process each raining area. Fig. 8 summarises the steps taken in processing the data with the boxes numbered 1-7 indicating different steps in the process. First a mask is generated to separate the raining and non-raining pixels (Fig. 8, Box 1) in the instantaneous radar image; pixels below a threshold of $1 \mathrm{~mm} / \mathrm{hr}$ are considered as non-raining and the remaining pixels are marked as raining. 

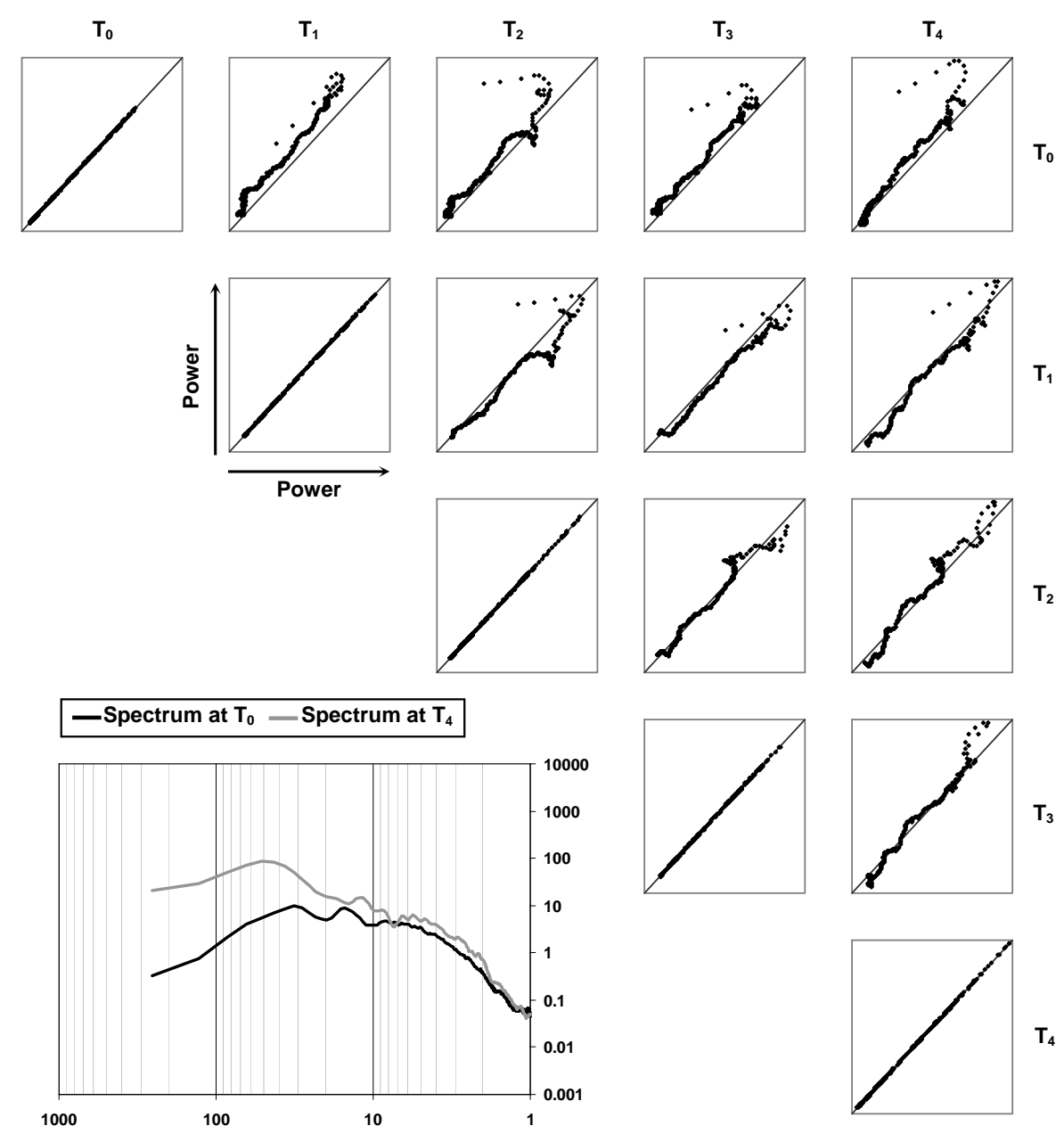

Fig. 12. Spectral persistence scatter plots of the sequence of 1 st IMSs of each pair of rainfall fields $T_{0}, \cdots, T_{4}$.

An outer boundary border-tracing algorithm (Sonka et al., 1999 ) is used to establish a boundary "fence" around each raining area (Fig. 8, Box 2) and a flood-fill procedure is then used to fill each raining area with a unique identifier, resulting in separately labelled raining regions (Fig. 8, Box 3). To reduce the computational burden of the algorithm even further, the boundary "fence" is decimated by a factor of 5 to reduce the continuous string of border points to "fence posts" while retaining the gross shape of the raining areas (Fig. 8, Box 4). The next step in the processing of the data is to isolate the extrema in the rainfall field (Fig. 8, Boxes 5 and 6). There are numerous possible techniques for identifying extrema in the rainfall field. Nunes et al. (2003) use a morphological reconstruction technique. One alternative, which was explored, is based on image segmentation and detection of extremal plateaus. However, our method of choice was to use a simple 8 neighbour search routine for identification of pixels with extreme values as done by Linderhed (2004). The choice was partly for convenience, but also because the majority of the (non-zero) extreme values in the rainfall fields studied turned out to consist of single pixels. There is a rich literature on image processing techniques and the reader is referred to an introductory text such as Sonka et al. (1999) to explore the field further. Finally, the EMD analysis is carried out using the extrema within each raining area and the zeros at the "fence posts" of non-raining border pixels to specify the extremal envelopes (Fig. 8, Box 7). Only one step of decomposition is shown here - the data is decomposed into the noisy first IMS and the first residual.

\subsection{Results}

An analysis of over 800 individual radar scans, embodying mixtures of various ratios of Stratiform and Convective rainfall types, was carried out to determine the effectiveness of the 2-D EMD algorithm in separating the high frequency spatial components from the original rainfall data. Working on the basis that the average characteristics of the data over a range of spatial scales summarised by the power spectrum is intuitively useful, the (radially averaged) power spectra 

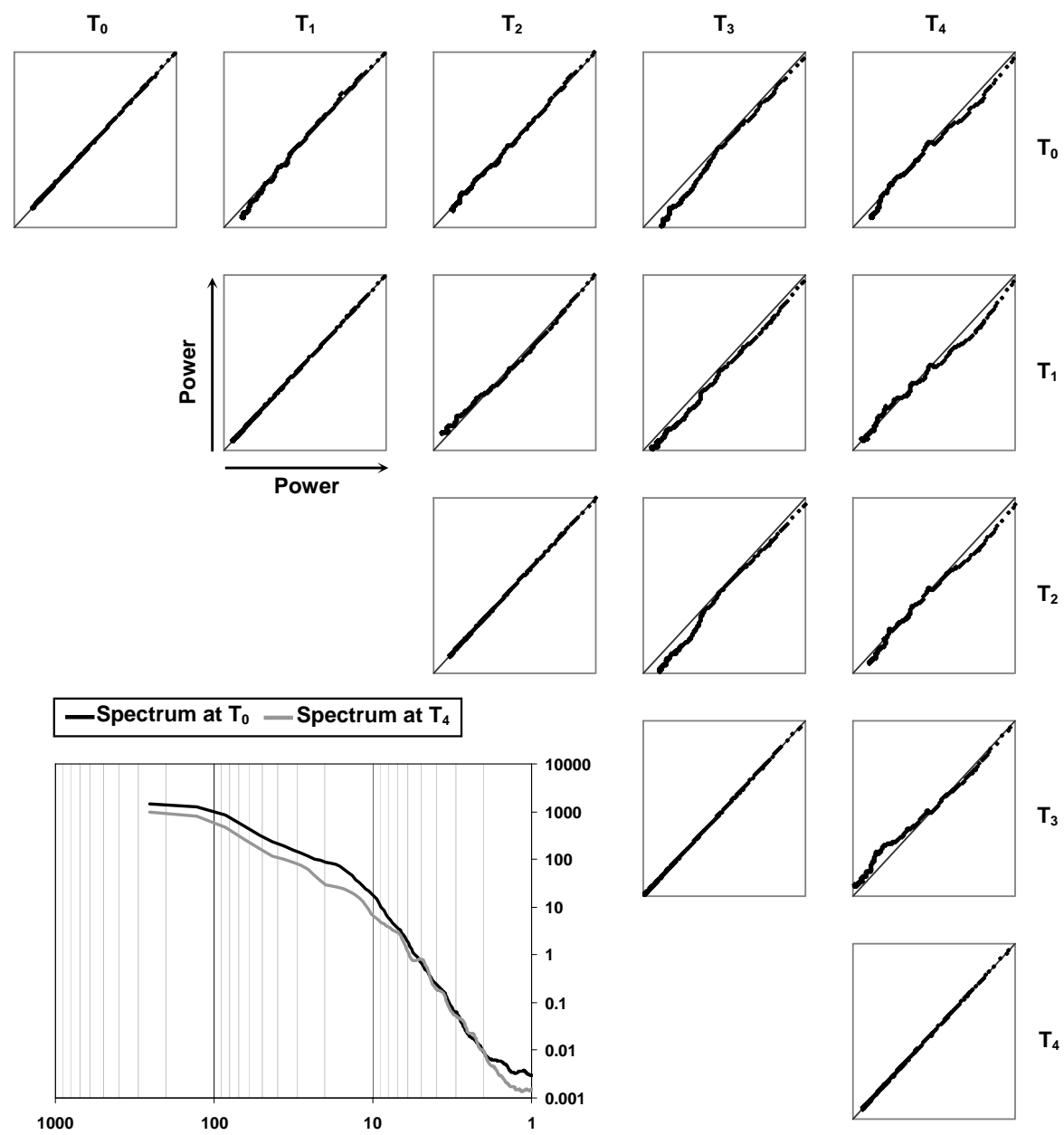

Fig. 13. Spectral persistence scatter plots of the sequence of 1 st Residual of each pair of rainfall fields $T_{0}, \cdots, T_{4}$.

of (i) the original data, (ii) the first IMS and (iii) the first residual of each image were examined and compared. Fig. 9 shows a typical result; the power spectrum of the residual shows a very close correspondence with that of the original data at high wavelengths while it contains far less power at the lower wavelengths. In contrast, the spectrum of the first (noisy) IMS has very little power relative to the data's spectrum at high wavelengths but shows a strong correspondence at the lowest wavelengths. Fig. 9 clearly indicates how the 2-D EMD technique moves the bulk of the high frequency components in the original data into the first IMS and leaves the high power, lower frequencies in the residual. Fig. 10 shows a time average of this behaviour by plotting the mean values at each wavelength of the three spectra over five consecutive radar scans (beginning with the data used to produce Fig. 9). The radar scans are captured at approximately fiveminute intervals. It is interesting to observe that the average of the spectra of the first IMSs is flat for wavelengths longer than $10 \mathrm{~km}$, suggesting nearly white noise over this range.
The temporal persistence exhibited at the spatial scales represented in each of the three sequences of: (i) the data, (ii) the first IMS and (iii) the first residual was examined by considering their temporally consecutive power spectra. The notion of "spectral persistence" was used to determine how variable the spatial structure (at a particular spatial scale) is in time and hence to give an indication of the temporal predictive capability at each spatial scale. A summarised example of the analysis of a sequence of 5 radar rainfall images is presented in Figs. 11, 12 and 13 where a "matrix" of scatter plots is shown in each case. Scatter-plots of the pairs of power values at each discrete wavelength for five consecutive spectra (with the 1:1 line indicated) are shown for, the original data (Fig. 11) the first IMS (Fig. 12) and the first residual (Fig. 13). The rows and columns of the scatter-plot matrices are labelled from $T_{0}$ to $T_{4}$ and indicate separate radar scans between time $T=0$ and time $T=4$. Each block in the scatter-plot matrix represents a scatter-plot of the power at each wavelength for the spectrum computed at $T_{i}$ versus that of the spectrum computed at $T_{j}$. Clearly the plots on the 
"matrix" diagonal each compare a spectrum to itself and a perfect 1:1 relationship is observed in this case. For the offdiagonal plots, the degree of scatter amongst the data points indicates the degree of similarity between the spectra at individual wavelengths at increasing time lags with a large scatter indicating a weak similarity. The trends shown here are typical of the data analysed and show how the first (high average frequency) IMS has a temporally incoherent spatial structure, while the first (low average frequency) residual shows a temporally consistent structure. The behaviour shown in Figs. 9-13 suggests that the high frequency IMS components in spatial rainfall data do not contain much predictive capability, supporting the suggestions of Seed (2003) and Turner et al. (2004) to increase the degree of spatial smoothing and rely more on the information contained in the lower frequency components as forecast lead times increase.

\section{Conclusions}

A new technique for analysing the spatial scaling structure of rainfall fields has been presented. The technique is a two dimensional extension of Empirical Mode Decomposition for the analysis of non-linear and non-stationary time series. An EMD analysis in two dimensions linearly decomposes the spatially distributed rainfall data into a set of Intrinsic Mode Surfaces, which are approximately mutually orthogonal and sum back to the original data. Each IMS contains an oscillatory mode inherent in the data at a different (narrow) range of spatial frequencies. The EMD analysis successively extracts the IMS with the highest local spatial frequencies in a recursive way, which is effectively a set of successive lowpass spatial filters based entirely on the properties exhibited by the data. The utility of the EMD technique for signal separation has been demonstrated in both one and two dimensions and applied to the analysis of a large set of 800 radar rainfall images in South Africa. The 2-D EMD technique is proposed here in the context of rainfall nowcasting to separate the less persistent high frequency components from the more persistent low frequency ones in the data. The aim is to remove the noisy high frequency components, which do not exhibit a strong temporal correlation and add little structural information to nowcasting algorithms. The scale separation achieved by 2-D EMD has been analysed using radially averaged power spectra to summarise the spatial structure of the data and filter outputs. In addition these power spectra have also been used to examine the temporal persistence of the spatial structure exhibited by the first IMS and it's residual. The results presented in this paper agree with other work in the hydrometeorological literature, which suggests that the low frequency spatial components in rainfall data are most useful in a nowcasting context. This methodology is being exploited in ongoing research into rainfall nowcasting.
Acknowledgements. The authors would like to thank the Water Research Commission of South Africa for supporting the work presented herein through contracts K5/1249 and K5/1425. The contributions of two anonymous reviewers were also helpful in improving the submission.

Edited by: M. Sivapalan

\section{References}

Balocchi, R., Menicucci, D., Santarcangelo, E., Sebastiani, L., Gemignani, A., Gellarducci, B., and Varanini, M.: Deriving the respiratory sinus arrhythmia from the heartbeat time series using empirical mode decomposition, Chaos, Solitons and Fractals, 20, 1, 171-177, 2004.

Daubechies I.: Ten Lectures on Wavelets, Society for Industrial and Applied Mathematics, Philadelphia, Pennsylvania, 1992.

Chiew, F. H. S., Peel M. C., Amirthanathan, G. E., and Pegram, G. G. S.: Identification of oscillations in historical global streamflow data using empirical mode decomposition. Regional Hydrological Impacts of Climatic Change - Hydroclimatic Variability, Proceedings of symposium S6 held during the Seventh IAHS Scientific Assembly at Foz do Iguaçu, Brazil, IAHS Publ. 296, 53-62, 2005.

Chiles, J. P. and Delfiner P.: Geostatistics: Modelling spatial uncertainty, Wiley, 1999.

Flandrin, P., Rilling, G., and Goncalves, P.: Empirical Mode Decomposition as a filter bank, IEEE Signal Processing Letters, 11, 2, 112-114, 2004.

Gloersen, P. and Huang, N.: Comparison of Interannual Intrinsic Modes in Hemispheric Sea Ice Covers and Other Geophysical Parameters, IEEE Transactions on Geoscience and Remote Sensing, 41, 5, 1062-1074, 2003.

Han, C., Guo, H., Wang, C., and Fan, D.: A novel method to reduce speckle in SAR images, Int. J. Remote Sensing, 23, 23, 50955101, 2002.

Harris, D., Foufoula-Georgiou, E., Droegemeier, K. K., and Levit, J. J.: Multiscale Statistical Properties of a High-Resolution Precipitation Forecast, J. Hydrometeor., 2, 406-418, 2001.

Huang, N. E., Shen, Z., Long, S. R., Wu, M. C., Shih, H. H., Zheng, Q., Yen, N., Tung, C. C., and Liu, H. H.: The Empirical Mode Decomposition and the Hilbert Spectrum for Nonlinear and NonStationary Time Series Analysis, Proceedings: Mathematical, Physical and Engineering Sciences, Royal Society London, 454, 1971, 903-995, 1998.

Huang, N. E., Shen, Z., and Long, S. R.: A new view of Nonlinear water waves: the Hilbert Spectrum, Ann. Rev. Fluid Mech., 31, 417-457, 1999.

Hwang, P. A., Huang, N. E., and Wang, D. W.: A note on analyzing nonlinear and nonstationary ocean wave data, App. Ocean Res., 25, 4, 187-193, 1999.

Linderhed, A.: 2-D empirical mode decompositions - in the spirit of image compression, Proceedings of SPIE, Wavelet and Independent Component Analysis Applications IXI, Volume 4738, 2002.

Nunes, J. C., Bouaoune, Y., Delechelle, E., Niang, O., and Bunel, P.: Image analysis by bidimensional empirical mode decomposition, Image and Vision Computing, 21, 1019-1026, 2003. 
Pegram, G. G. S. and Clothier, A. N.: High Resolution Space-Time Modelling of Rainfall: The "String of Beads" Model, J. Hydrol., 241, 26-41, 2001.

Press, W. H., Teukolsky S. A., Vetterling W. T., and Flannery, B. P.: Numerical Recipes in $\mathrm{C}-\mathrm{The}$ art of scientific computing, Second Edition, Cambridge University Press, 1992.

Schertzer, D. and Lovejoy, S.: Physical Modelling and Analysis of Rain and Clouds by Anisotropic Scaling Multiplicative Processes, J. Geophys. Res., 92, D8, 9693-9714, 1987.

Seed, A. W.: A Dynamic and Spatial Scaling Approach to Advection Forecasting, J. Appl. Meteor., 42, 381-388, 2003.

Sonka, M., Hlavac, V., and Boyle, R.: Image Processing, Analysis and Machine Vision, 2nd Edition, PWS Publishing, Pacific Grove, 1999.
Turner, B. J., Zawadzki, I., and Germann, U.: Predictability of precipitation from continental radar images. Part III: Operational nowcasting implementation (MAPLE), J. Appl. Meteor., 43, 231-248, 2004.

Wesson, S. M. and Pegram, G. G. S.: Radar rainfall image repair techniques, Hydrol. Earth Syst. Sci., 8, 220-234, 2004,

SRef-ID: 1607-7938/hess/2004-8-220.

Zhang, R. R., Ma, S., and Hartzell, S.: Signatures of the seismic source in EMD-based characterization of the 1994 Northridge, California, earthquake recordings, Bulletin of the Seismological Society of America, 93, 1, 501-518, 2003. 